\title{
Chapter 5 \\ Theoretical Approaches: Exciton Theory, Coulomb Interactions and Fluctuation-Dissipation Theorem
}

In this chapter, we introduce the main framework for Förster-type nonradiative energy transfer; starting from exciton theory, going through Coulomb interaction, and finalizing with the fluctuation-dissipation theorem. Part of this chapter is reprinted (adapted) with permission from Ref. [1]. Copyright 2013 American Physical Society.

\subsection{Electron-Hole Interaction (Exciton)}

An exciton is a quasiparticle consisting of a bound state of an electron and a hole interacting via Coulomb force. An exciton can move through the medium (e.g., semiconductor crystal) and transport energy; and since an exciton is electrically neutral, it does not transport charge. An exciton can be created by external excitation, for example, through the absorption of a photon, with $E \geq E_{g}$. In this direct process, an electron is excited from the valence band to the conductive band, leaving behind a hole with opposite charge in the valence band, to which the electron can bind due to the attractive Coulombic interaction. Because of the Coulombic attraction between the electron and the hole in an exciton, the internal states are analogous to those of the hydrogen atom, and some of the lower energy states lie below the conduction band by an energy equivalent to the exciton binding energy in that state (Figs. 5.1 and 5.2).

An exciton has two quantities: (1) the pseudomomentum of the electron-hole pair and (2) the relative momentum of the electron and the hole. The pseudomomentum, which is equal to the vector sum of the individual momenta of the electron and the hole, enables an exciton to move throughout a crystal; and the relative momentum 


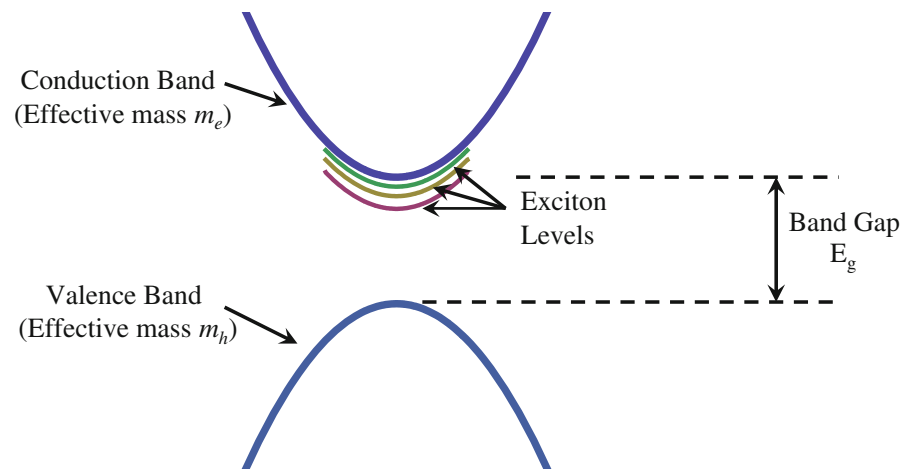

Fig. 5.1 Exciton levels for a simple band structure at $k=0$

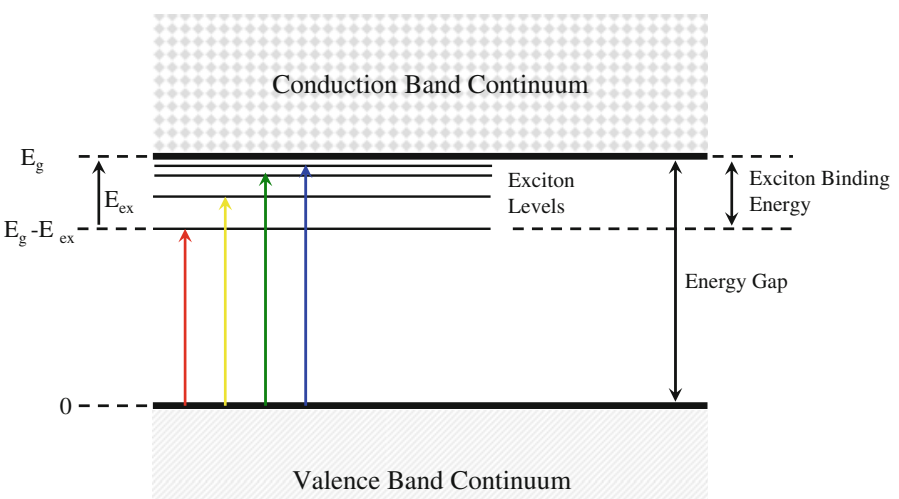

Fig. 5.2 Energy levels of an exciton created in a direct process. Optical transitions are shown by arrows

determines its internal structure. Excitons are classified into (1) a tightly bound exciton (Frenkel exciton) and (2) a weakly bound exciton (Mott-Wannier exciton).

\subsubsection{Frenkel Excitons}

In a tightly bound exciton the excitation is localized on a single atom (Fig. 5.3), i.e., a Frenkel exciton is an excited state of a single atom. A Frenkel exciton can hop from one atom to another via coupling between neighbors. Similar to all other excitation in a periodic structure, the translational states of Frenkel excitons take the form of propagating waves. 
Fig. 5.3 Schematic illustration of a tightly-bound exciton (Frenkel exciton) localized on one atom in a crystal

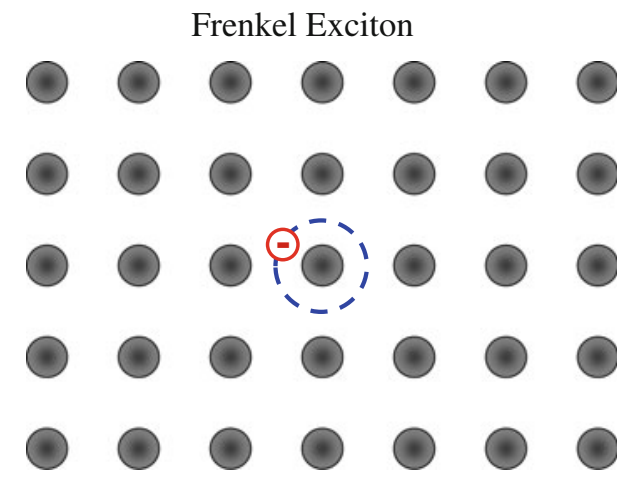

Consider a crystal of $N$ atoms on a line or ring. If $u_{j}$ is the ground state of atom $j$, the ground state of the crystal is [2]

$$
\psi_{g}=u_{1} u_{2} \cdots u_{j} \cdots u_{N-1} u_{N}
$$

If a single atom $j$ is in an excited state $v_{j}$, the system is described by

$$
\phi_{j}=u_{1} u_{2} \cdots u_{j-1} v_{j} u_{j+1} \cdots u_{N-1} u_{N}
$$

If we consider that the excited atom interacts only with nearby atoms in its ground state, then the excitation will be passed from atom to atom.

Applying the Hamiltonian of the system on the function $\phi_{j}$ with the $j$ th atom excited, we obtain the following

$$
H \phi_{j}=\varepsilon \phi_{j}+T\left(\phi_{j-1}+\phi_{j+1}\right)
$$

where $\varepsilon$ is the free atom excitation energy; $T$ is the transfer rate of the excitation from $j$ to its nearest neighbors, $j-1$ and $j+1$. The solutions of (5.3) are the waves of the Bloch form:

$$
\psi_{k}=\sum_{j} \exp (i j k a) \phi_{j}
$$

Operating the Hamiltonian on (5.4)

$$
H \psi_{k}=\sum_{j} e^{i j k a} H \phi_{j}=\sum_{j} e^{i j k a}\left[\varepsilon \phi_{j}+T\left(\phi_{j-1}+\phi_{j+1}\right)\right]
$$

Rearranging the right-hand side of (5.5) 


$$
H \psi_{k}=\sum_{j} e^{i j k a}\left[\varepsilon+T\left(e^{i k a}+e^{-i k a}\right)\right] \phi_{j}=(\varepsilon+2 T \cos (k a)) \psi_{k}
$$

so that the energy eigenvalues are (Fig. 5.4):

$$
E_{k}=\varepsilon+2 T \cos (k a)
$$

Applying the periodic boundary conditions, the allowed values of the wavevector $k$ are:

$$
k=\frac{2 \pi n}{N a} ; \quad n=-\frac{1}{2} N,-\frac{1}{2} N+1, \ldots, \frac{1}{2} N-1
$$

\subsubsection{Mott-Wannier Excitons}

In a weakly bound exciton, the electron-hole distance is larger than the lattice constant of the crystal, meaning that the exciton is delocalized over several atoms (Fig. 5.5). The Mott-Wannier exciton is similar to the hydrogen atom problem. In other words, the Mott-Wannier exciton can be treated as a two-particle system weakly interacting, in which case the electron and hole energy (at $\mathbf{k}=0$ ) is given by $[3,4]$

$$
\varepsilon_{c}(\mathbf{k})=\varepsilon_{c}(0)+\frac{\hbar^{2} k^{2}}{2 m_{e}^{*}}
$$

Fig. 5.4 $E-k$ diagram for a Frenkel exciton

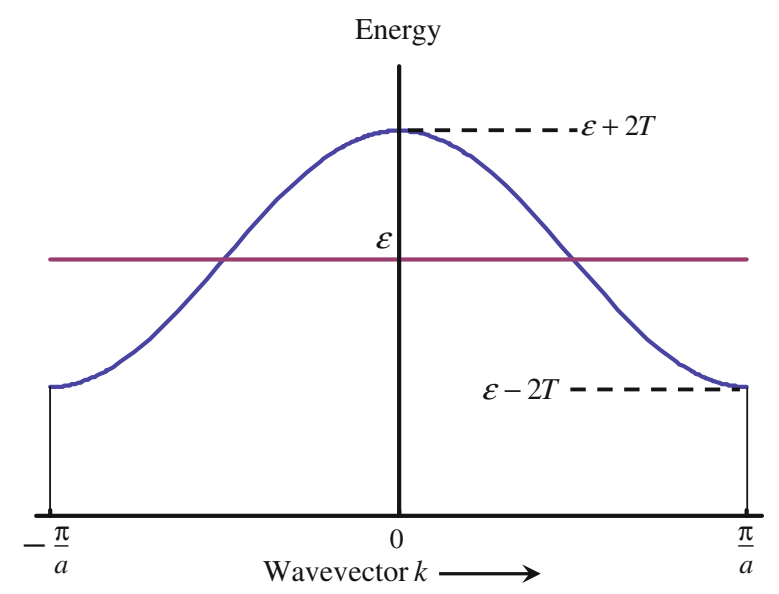




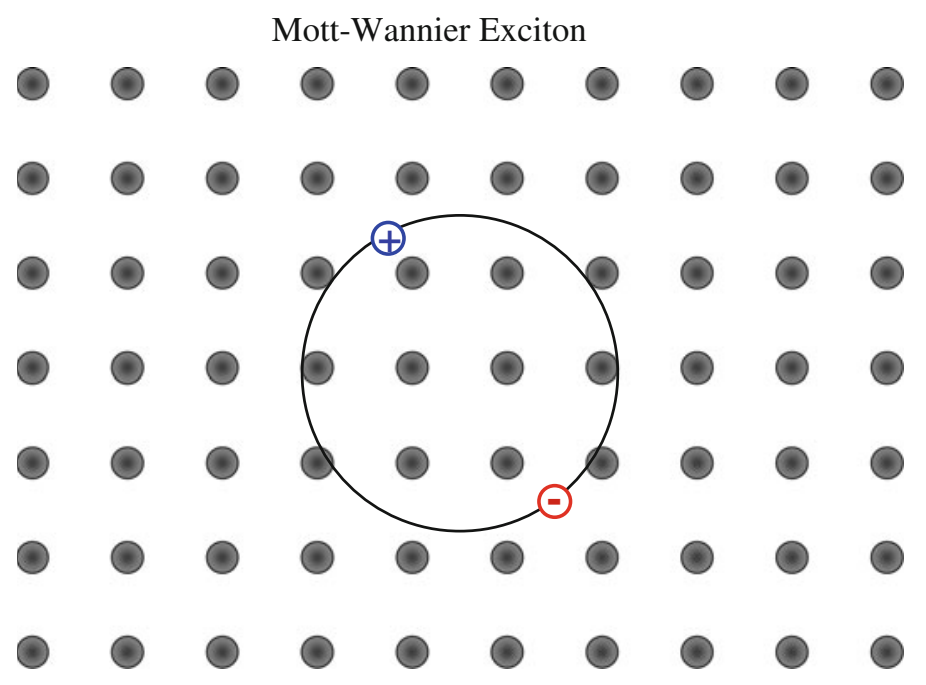

Fig. 5.5 Schematic illustration of a weakly bound exciton (Mott-Wannier exciton) delocalized over several atoms in the crystal

And

$$
\varepsilon_{v}(\mathbf{k})=\varepsilon_{v}(0)-\frac{\hbar^{2} k^{2}}{2 m_{h}^{*}}
$$

where $m_{e}^{*}$ and $-|e|$ are the electron mass and charge; and $m_{h}^{*}$ and $+|e|$ are the hole mass and charge, respectively. For simplicity, we assume that the crystal has simple valence and conduction bands.

The total kinetic energy is

$$
\mathbf{P}=\frac{\mathbf{p}_{e}^{2}}{2 m_{e}^{*}}+\frac{\mathbf{p}_{h}^{2}}{2 m_{h}^{*}}
$$

where $\mathbf{p}_{e}^{2}$ and $\mathbf{p}_{h}^{2}$ are the electron and hole momenta, respectively. The effective Hamiltonian for the two-particle system when interacting in a dielectric medium of relative dielectric constant $\varepsilon$ is

$$
H_{e f f}=-\frac{\hbar^{2}}{2 m_{e}^{*}} \nabla_{e}^{2}-\frac{\hbar^{2}}{2 m_{h}^{*}} \nabla_{h}^{2}-\frac{1}{4 \pi \varepsilon_{0} \varepsilon} \frac{e^{2}}{\left|\mathbf{r}_{e}-\mathbf{r}_{h}\right|}
$$

The solution for this Hamiltonian is 


$$
E_{n}=E_{g}-\frac{1}{\left(4 \pi \varepsilon_{0}\right)^{2}} \frac{\mu_{e x} e^{4}}{2 \hbar^{2} \varepsilon^{2}} \frac{1}{n^{2}}+\frac{\hbar^{2} \mathbf{K}^{2}}{2 M^{*}}
$$

where $E_{n}$ is the exciton energy, $E_{g}=\varepsilon_{c}(0)-\varepsilon_{v}(0)$ is the bandgap energy, $\frac{1}{\mu_{e x}}=$ $\frac{1}{m_{e}^{*}}+\frac{1}{m_{h}^{*}}$ is the reduced exciton mass, and $M^{*}=m_{e}^{*}+m_{h}^{*}$ is the effective exciton mass. A useful parameter for an exciton is the exciton Bohr radius $\left(a_{e x}\right)$. It is obtained from the second term of (5.13). Therefore, the exciton Bohr is given by

$$
a_{e x}=4 \pi \varepsilon_{0} \frac{\hbar^{2} \varepsilon}{\mu_{e x} e^{2}} n^{2}
$$

\subsection{Coulombic Interaction}

In both cases, the Coulombic interaction between the electron and the hole is treated with standard second order perturbation [5].

$$
E=E_{0}+\lambda\left\langle 0\left|H^{\prime}\right| 0\right\rangle+\lambda^{2} \sum_{i} \frac{\left|\left\langle 0\left|H^{\prime}\right| i\right\rangle\right|^{2}}{E_{0}-E_{i}}
$$

where $E_{0}$ and $|0\rangle$ are the unperturbed eigenenergy and eigenvector of the e-h ground state based on a kinetic energy, $H^{\prime}$ is the perturbation Hamiltonian, $E_{i}$ and $|i\rangle$ are the unperturbed eigenenergy and eigenvector of all the other states. The effective Coulombic interaction is given by

$$
H^{\prime}\left(\mathbf{r}_{e}, \mathbf{r}_{h}\right)=\lambda^{-1} V\left(\mathbf{r}_{\alpha}, \mathbf{r}_{\beta}\right)
$$

where $V\left(\mathbf{r}_{\alpha}, \mathbf{r}_{\beta}\right)$ is the potential function, which depends on $R_{N C}$, overall nanocrystal (NC) radius, and $\varepsilon=\frac{\varepsilon_{N C}}{\varepsilon_{M}}$ with $\varepsilon_{N C}$ and $\varepsilon_{M}$ being the NC and surrounding medium dielectric constants, respectively.

$$
\begin{aligned}
& V\left(\mathbf{r}_{\alpha}, \mathbf{r}_{\beta}\right)=\frac{1}{4 \pi \varepsilon_{0} \varepsilon_{N C}} \sum_{\alpha, \beta} q_{\alpha} q_{\beta}\left\{\frac{1}{\left|\mathbf{r}_{\alpha}-\mathbf{r}_{\beta}\right|}+\frac{(\varepsilon-1)}{R_{N C}}\right. \\
& {\left.\left[\sum_{i=1}^{\infty} \frac{\left(r_{\alpha} r_{\beta}\right)^{i}}{1+\varepsilon\left(\frac{i}{i+1}\right)} P_{l}\left(\frac{\mathbf{r}_{\alpha} \cdot \mathbf{r}_{\beta}}{r_{\alpha} r_{\beta}}\right)+\frac{1}{2} \sum_{j=1}^{\infty} \frac{\left(r_{\alpha}\right)^{2 j}+\left(r_{\beta}\right)^{2 j}}{1+\varepsilon\left(\frac{j}{j+1}\right)}\right]\right\} }
\end{aligned}
$$

Thus, $E_{X}$ energy is given by 


$$
\begin{gathered}
E_{X}=E_{0}^{e}+E_{0}^{h}+\lambda\left\langle 0,0\left|H^{\prime}\right| 0,0\right\rangle+\lambda^{2} \sum_{\alpha, \beta} \frac{\left|\left\langle 0,0\left|H^{\prime}\right| \alpha, \beta\right\rangle\right|^{2}}{\left(E_{\alpha}^{e}-E_{0}^{e}\right)+\left(E_{\beta}^{h}-E_{0}^{h}\right)} \\
E_{X}=E_{0}^{e}+E_{0}^{h}-\lambda V_{00}-\lambda^{2} \sum_{\alpha, \beta} \frac{\left|V_{\alpha \beta}\right|^{2}}{\left(E_{\alpha}^{e}-E_{0}^{e}\right)+\left(E_{\beta}^{h}-E_{0}^{h}\right)}
\end{gathered}
$$

with $\alpha \neq 0$ and $\beta \neq 0$ and $V_{\alpha \beta}$ is defined as

$$
V_{\alpha \beta}=\left\langle 0,0\left|H^{\prime}\right| \alpha, \beta\right\rangle
$$

\subsection{Exciton in Quantum Dots: Single-Particle Quantization Energy and Coulomb Interaction}

The aim is to determine for the particle in a spherical box problem the envelope wavefunction $\psi$ for electron and hole. We consider a two-band (valance and conduction) system (Fig. 5.6). The eigenfunctions of the hole and electron are written as a product of an envelope function $\varphi_{e, h}(\mathbf{r})$ and a lattice periodic function $u_{V, C}(\mathbf{r})[6]:$

$$
\begin{aligned}
& \psi_{e}(\mathbf{r})=\varphi_{e}(\mathbf{r}) u_{C}(\mathbf{r}) \\
& \psi_{h}(\mathbf{r})=\varphi_{h}(\mathbf{r}) u_{V}(\mathbf{r})
\end{aligned}
$$

Fig. 5.6 Quantum dot energy levels for the electron and hole in the conduction and valance band, respectively
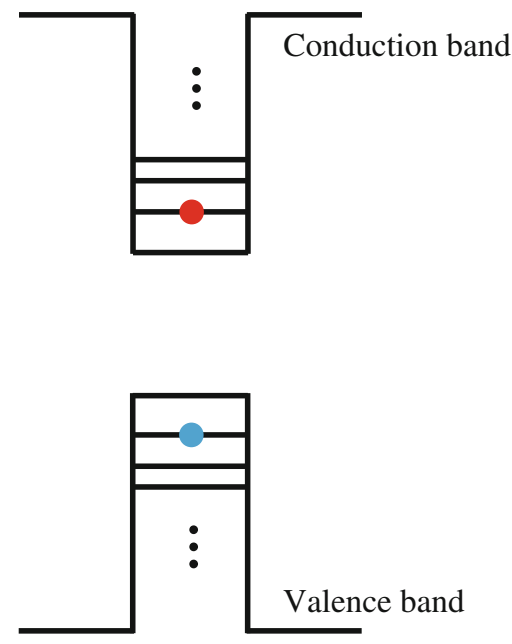
The envelope functions are the zero order eigenfunctions of the electron-hole pair Hamiltonian

$$
H_{e f f}=-\frac{\hbar^{2}}{2 m_{e}^{*}} \nabla_{e}^{2}-\frac{\hbar^{2}}{2 m_{h}^{*}} \nabla_{h}^{2}+V_{e}\left(\mathbf{r}_{e}\right)+V_{h}\left(\mathbf{r}_{h}\right)-\frac{1}{\varepsilon_{Q D}} \frac{e^{2}}{\left|\mathbf{r}_{e}-\mathbf{r}_{h}\right|}
$$

where $m_{e}^{*}, m_{h}^{*}$ is the electron and home effective mass, respectively; $\varepsilon_{Q D}$ is the static dielectric constant of the nanocrystals (quantum dot); and the confinement potentials are given as follows

$$
V_{e(h)}\left(r_{e(h)}\right)=\left\{\begin{array}{cc}
0 & \text { for } r_{e(h)}<R_{Q D} \\
\infty & \text { for } r_{e(h)}>R_{Q D}
\end{array}\right.
$$

Here $R_{Q D}$ is the quantum dot radius.

Note that the last term on the right hand side of (5.23) is the first order perturbation term. The orthonormal functions are zero outside of the dot, and inside it are given by

$$
\varphi_{n l m}^{e(h)}\left(\mathbf{r}_{e(h)}\right)=\sqrt{\frac{2}{R_{Q D}^{3}}} \frac{j_{l}\left(\chi_{n l} \frac{r}{R_{Q D}}\right)}{j_{l+1}\left(\chi_{n l}\right)} Y_{l m}(\theta, \phi)
$$

where $Y_{l m}(\theta, \phi)$ is the spherical harmonics functions, $j_{l}(x)$ is the spherical Bessel functions, and $\chi_{n l}$ are the spherical Bessel functions $n$ th-order zeros. The energy eigenvalues $E_{n l, n^{\prime} l^{\prime}}$, including the requirement that the wavefunction vanishes at $r=R_{Q D}$ and the first order perturbation term, are given by

$$
E_{n l, n^{\prime} l^{\prime}}=E_{g}+\frac{\hbar^{2}}{2 m_{e}^{*}}\left(\frac{\chi_{n l}^{2}}{R_{Q D}^{2}}\right)+\frac{\hbar^{2}}{2 m_{h}^{*}}\left(\frac{\chi_{n^{\prime} l^{\prime}}^{2}}{R_{Q D}^{2}}\right)-\frac{1.8 e^{2}}{\varepsilon_{Q D} R_{Q D}}
$$

Here, $E_{g}$ is the bulk bandgap.

\subsection{Fermi's Golden Rule and Fluctuation Dissipation Theorem}

In this section, we outline a macroscopic approach to the problem of dipole-dipole energy transfer. We restrict ourselves to the case of a single electron-hole pair (exciton) in the donor nanostructure. Moreover, we consider only two states $(|0\rangle-$ the ground state and $|e x c\rangle$-the excited state). These states are constructed using simplified wavefunctions, i.e., we consider excitonic states without mixing of the heavy- and light-hole states. Furthermore, the spin part is not included in our model. 
FRET is a directional process initiated by an absorbed photon in a donor that creates an exciton in a higher excited state, relaxing very fast to the first excited state by higher order processes. This exciton can subsequently be either recombined (through radiative or nonradiative means) or transferred to an acceptor because of the Coulomb interaction between dipoles in the D-A pair. If the exciton is transferred, it will occupy a higher excited state in the acceptor and relax (very fast) to its first excited state to finally recombine through a radiative or nonradiative process. Note that FRET occurs only when the donor possesses a greater or equal bandgap compared to the acceptor. Figure 5.7 shows the energy diagram for this process.

The probability of an exciton transfer from the donor to the acceptor is given by the Fermi's Golden Rule (5.27).

$$
\gamma_{\text {trans }}=\frac{2}{\hbar}\left\{\sum_{f}\left|\left\langle f_{\text {exc }} ; 0_{\text {exc }}\left|\hat{V}_{\text {int }}\right| i_{\text {exc }} ; 0_{\text {exc }}\right\rangle\right|^{2} \delta\left(\hbar \omega_{\text {exc }}-\hbar \omega_{f}\right)\right\}
$$

where $\left|i_{\text {exc }} ; 0_{\text {exc }}\right\rangle$ is the initial state with an exciton in the donor and zero exciton in the acceptor; $\left|f_{\text {exc }} ; 0_{\text {exc }}\right\rangle$ is the final state with an exciton in the acceptor and zero exciton in the donor; $\hat{V}_{\text {int }}$ is the exciton Coulomb interaction operator; and $\hbar \omega_{\text {exc }}$ is the exciton's energy. Neglecting the coherent coupling between excitons, i.e., the initial and final states can be written as $\left|i_{\text {exc }} ; 0_{\text {exc }}\right\rangle=\left|i_{\text {exc }}\right\rangle\left|0_{\text {exc }}\right\rangle$ and $\left|f_{\text {exc }} ; 0_{\text {exc }}\right\rangle=\left|f_{\text {exc }}\right\rangle\left|0_{\text {exc }}\right\rangle$, and the Fermi's Golden Rule can be approximated by

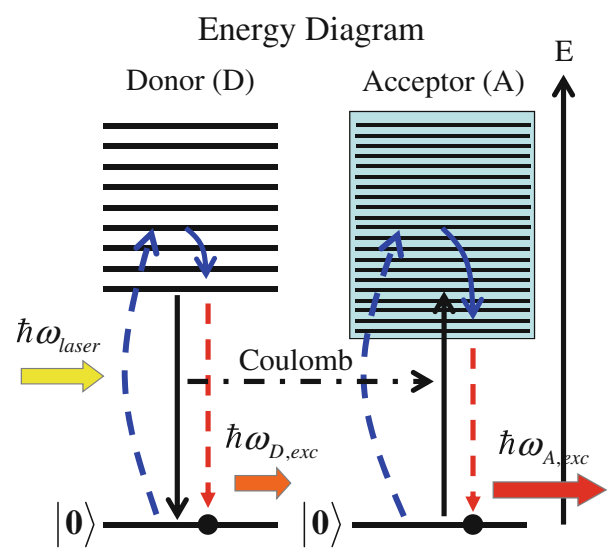

Fig. 5.7 Energy diagram for the directional process of exciton transfer from the donor to the acceptor. Blue dash lines represent the absorption process of the nanostructure (donor/acceptor). Blue solid lines denote fast relaxation process. Red dash lines illustrate light emission process (relaxation from the lowest excited state to ground state). Black solid lines represent the energy transfer from the donor to the acceptor. Horizontal solid black line illustrates the Coulomb interaction between the donor and the acceptor [reprinted (adapted) with permission from Ref. 9 (Copyright 2008 American Physical Society)] 


$$
\begin{array}{r}
\gamma_{\text {trans }}=\frac{2}{\hbar}\left\{\sum_{f}\left|\left\langle f_{\text {exc }} ; 0_{\text {exc }}\left|\hat{V}_{\text {int }}\right| i_{\text {exc }} ; 0_{\text {exc }}\right\rangle\right|^{2} \delta\left(\hbar \omega_{\text {exc }}-\hbar \omega_{f}\right)\right\} \\
\gamma_{\text {trans }} \approx \frac{2}{\hbar}\left\{\sum_{f}\left|\left\langle f_{\text {exc }}\left|\hat{U}_{\text {int }}\right| 0_{\text {exc }}\right\rangle\right|^{2} \delta\left(\hbar \omega_{\text {exc }}-\hbar \omega_{f}\right)\right\}
\end{array}
$$

where $\hat{U}_{\text {int }}=\left\langle 0_{\text {exc }}\left|\hat{V}_{\text {int }}\right| i_{e x c}\right\rangle$ is the potential energy created by the exciton. With the help of the fluctuation dissipation theorem (FDT) [7] and the formalism given in elsewhere [8,9], the Fermi's Golden Rule can be simplified into

$$
\begin{gathered}
\gamma_{\text {trans }} \approx \frac{2}{\hbar}\left\{\sum_{f}\left|\left\langle f_{\text {exc }}\left|\hat{U}_{\text {int }}\right| 0_{\text {exc }}\right\rangle\right|^{2} \delta\left(\hbar \omega_{\text {exc }}-\hbar \omega_{f}\right)\right\} \\
\gamma_{\text {trans }}=\frac{2}{\hbar}\left\{\frac{1}{2 \pi} \int_{-\infty}^{\infty} \exp \left(i \omega_{\text {exc }} t\right)\left\langle 0_{\text {exc }}\left|\hat{U}_{\text {int }}(t) \hat{U}_{\text {int }}(0)\right| 0_{\text {exc }}\right\rangle d t\right\} \\
\gamma_{\text {trans }}=-\frac{2 \pi}{\hbar}\left\{\frac{1}{\pi} \operatorname{Im}\left[F_{\text {exc }}\left(\omega_{\text {exc }}\right)\right]\right\}
\end{gathered}
$$

where $F_{e x c}\left(\omega_{e x c}\right)$ is the response function given by

$$
F_{\text {exc }}\left(\omega_{\text {exc }}\right)=\int d V \rho(\mathbf{r}) \Phi_{\text {int }}(\mathbf{r})
$$

Here $\rho(\mathbf{r})$ is the local non-equilibrium charge density and $\Phi_{\text {int }}(\mathbf{r})$ the effective electric potential created by the exciton. Since the charge density is given by $\nabla \cdot(\varepsilon(\mathbf{r}, \omega) \mathbf{E}(\mathbf{r}, \omega))=4 \pi \rho(\omega)$, the response function can be written as

$$
F_{\text {exc }}\left(\omega_{\text {exc }}\right)=\int d V \rho(\mathbf{r}) \Phi_{\text {int }}(\mathbf{r})=-\int d V\left(\frac{\varepsilon_{A}(\omega)}{4 \pi}\right) \mathbf{E}_{i n}(\mathbf{r}) \cdot \mathbf{E}_{i n}^{*}(\mathbf{r})
$$

where $\varepsilon_{A}(\omega)$ is the dielectric function of the acceptor and $\mathbf{E}_{i n}(\mathbf{r})$ is the electric field inside the acceptor, induced by an exciton in the donor. Finally, the energy transfer rate from the donor to the acceptor is given by

$$
\gamma_{\text {trans }}=\frac{2}{\hbar} \operatorname{Im}\left[\int d V\left(\frac{\varepsilon_{A}(\omega)}{4 \pi}\right) \mathbf{E}_{\text {in }}(\mathbf{r}) \cdot \mathbf{E}_{\text {in }}^{*}(\mathbf{r})\right]
$$

where $\mathbf{E}_{i n}(\mathbf{r})$ includes the effective electric field created by an exciton in the donor. Here, the electric field is calculated by 


$$
\mathbf{E}(\mathbf{r})=-\nabla \Phi(\mathbf{r})
$$

The electric potential, $\Phi(\mathbf{r})$, which is needed to compute $\gamma_{\text {trans }}$ (5.35), should be expressed as a total potential created by the electric potential of an exciton (on the donor side)

$$
\Phi_{\alpha}(\mathbf{r})=\left(\frac{e d_{e x c}}{\varepsilon_{e f f_{D}}}\right) \frac{\left(\mathbf{r}-\mathbf{r}_{0}\right) \cdot \hat{\alpha}}{\left|\mathbf{r}-\mathbf{r}_{0}\right|^{3}}
$$

where $e d_{e x c}$ is the dipole moment of the exciton and $\varepsilon_{e f f_{D}}$ is the effective dielectric constant of the donor, which depends on the geometry and the exciton dipole direction, $\alpha=x, y, z$. Note that, to estimate the FRET rate, we need to calculate the effective electric potential due to an exciton in the donor in the vicinity of an acceptor.

The average FRET rate is calculated as

$$
\gamma_{\text {trans }}=\frac{\gamma_{x, \text { trans }}+\gamma_{y, \text { trans }}+\gamma_{z, \text { trans }}}{3}
$$

where $\gamma_{\alpha, \text { trans }}$ is the transfer rate for the $\alpha$-exciton $(\alpha=x, y, z)$.

\section{References}

1. P.L. Hernández-Martínez, A.O. Govorov, H.V. Demir, Generalized theory of Förster-type nonradiative energy transfer in nanostructures with mixed dimensionality. J. Phys. Chem. C 117, 10203-10212 (2013)

2. C. Kittel, Introduction to solid state physics, 8th edn. (Wiley, New York, 2005)

3. P.Y. Yu, M. Cardona, Fundamentals of semiconductors: physics and materials properties, 3rd edn. (Springer, New York, 2005)

4. D.L. Dexter, R.S. Knox, Excitons (Interscience Publishers, Geneva, 1965)

5. L. Banyai, S.W. Koch, Semiconductor quantum dots (World Scientific Press, Singapore, 1993)

6. U. Woggon, Optical properties of semiconductors quantum dots (Springer, Germany, 1997)

7. P.M. Platzman, P.A. Wolf, Waves and interactions in solid state plasma (Academic Press, New York, 1973)

8. A.O. Govorov, J. Lee, N.A. Kotov, Theory of plasmon-enhanced Förster energy transfer in optically excited semiconductor and metal nanoparticles. Phys. Rev. B 76, 125308/1$125308 / 16(2007)$

9. P.L. Hernández-Martínez, A.O. Govorov, Exciton energy transfer between nanoparticles and nanowires. Phys. Rev. B, 78, 035314/1-035314/7 (2008) 